\title{
Cost of Acute Kidney Injury in Hospitalized Patients
}

\author{
Samuel A. Silver, MD, MSc ${ }^{1,2 *}$, Jin Long, $\mathrm{PhD}^{1}$, Yuanchao Zheng, MS ${ }^{1}$, Glenn M. Chertow, MD, MPH ${ }^{1}$
}

${ }^{1}$ Division of Nephrology, Stanford University School of Medicine, Palo Alto, California; ${ }^{2}$ Division of Nephrology, University of Toronto, Toronto, Canada.

BACKGROUND: The economic burden of acute kidney injury (AKI) is not well understood.

OBJECTIVE: To estimate the effects of AKI on hospitalization costs and length of stay (LOS).

DESIGN: Using data from the 2012 National Inpatient Sample, we compared hospitalization costs and LOS with and without AKI. We used a generalized linear model with a gamma distribution and a log link fitted to AKI to adjust for demographics, hospital differences, and comorbidities.

SETTING: United States

PATIENTS: $29,763,649$ adult hospitalizations without endstage renal disease.

EXPOSURE: AKI determined using International Classification of Diseases, $9^{\text {th }}$ Revision, Clinical Modification (ICD-9$\mathrm{CM}$ ) diagnosis codes.

MEASUREMENTS: Hospitalization costs and LOS.

RESULTS: AKI was associated with an increase in hospital- ization costs of $\$ 7933$ (95\% confidence interval [Cl], \$7608$\$ 8258)$ and an increase in LOS of $3.2(95 \% \mathrm{Cl}, 3.2-3.3)$ days compared to patients without AKI. When adjusted for patient and hospital characteristics, the associated increase in costs was $\$ 1795(95 \% \mathrm{Cl}, \$ 1692-\$ 1899)$ and in LOS, it was 1.1 (95\% Cl, 1.1-1.1) days. Corresponding results among patients hospitalized with AKI requiring dialysis were $\$ 42,077$ $(95 \% \mathrm{Cl}, \$ 39,820-\$ 44,335)$ and $11.5(95 \% \mathrm{Cl}, 11.2-11.8)$ days and $\$ 11,016(95 \% \mathrm{Cl}, \$ 10,468-\$ 11,564)$ and $3.9(95 \%$ $\mathrm{Cl}, 3.8-4.1)$ days. AKI was associated with higher hospitalization costs than myocardial infarction and gastrointestinal bleeding, and costs were comparable to those for stroke, pancreatitis, and pneumonia.

CONCLUSIONS: In the United States, AKI is associated with excess hospitalization costs and prolonged LOS. The economic burden of AKI warrants further attention from hospitals and policymakers to enhance processes of care and develop novel treatment strategies. Journal of Hospital Medicine 2017;12:70-76. (c) Society of Hospital Medicine
Acute kidney injury (AKI) is a common complication that affects as many as $20 \%$ of hospitalized patients, depending on the definition employed. ${ }^{1-3}$ AKI is associated with significant morbidity and mortality; hospitalized patients with AKI require more investigations and medications, ${ }^{4}$ develop more postoperative complications, ${ }^{5}$ and spend more time in the intensive care unit than do patients without AKI. ${ }^{6}$ Inhospital mortality for patients with AKI has recently been estimated between $20-25 \%, 3$ and critically ill patients with AKI requiring dialysis experience mortality rates in excess of $50 \%{ }^{8,9}$ $\mathrm{AKI}$ and its accompanying complications may continue to rise, as the incidence of AKI and AKI requiring dialysis is increasing at a rate of approximately $10 \%$ per year. ${ }^{10-12}$

Owing to poor outcomes and rising incidence, AKI has emerged as a major public health concern with high human and financial costs; however, the costs related to AKI have been excluded from recent United States Renal Data System estimates. ${ }^{13}$ Most studies that have explored the costs related to hospitalizations complicated by AKI have been

\footnotetext{
*Address for correspondence and reprint requests: Samuel A. Silver, Stanford University School of Medicine, Division of Nephrology, 1070 Arastradero Road, Palo Alto, CA, 94304; Telephone: 650-504-0030; Fax: 650-721-1443; E-mail: sam.silver@utoronto.ca

Additional Supporting Information may be found in the online version of this article.
}

Received: May 9, 2016; Revised: July 13, 2016; Accepted: July 24, 2016

2017 Society of Hospital Medicine DOI 10.12788/jhm.2683 single-center or local studies in specialized patient populations. ${ }^{4,5,14-18}$ Very few studies have used data after the year 2000, when the incidence of AKI began to increase, likely related to a combination of patient age, comorbidity burden, sepsis, heart failure, and nephrotoxic medications. ${ }^{10,11}$ Moreover, it is unclear which patient and hospital characteristics contribute most to the cost of an AKI hospitalization, and how the costs of AKI compare to those for other acute medical conditions. Such information is important for hospitals, policymakers, and researchers to target prevention and management strategies for high-risk and high-cost patient groups.

The main objectives of this study were to determine the costs of AKI-related hospitalization, and patient and hospital factors associated with these costs. We hypothesized that costs related to AKI would add several thousand dollars to each hospitalization and would eclipse the cost of many higher profile acute medical conditions.

\section{METHODS}

\section{Study Population}

We extracted data from the National Inpatient Sample (NIS), a nationally representative administrative database of hospitalizations in the United States (U.S.) created by the Agency for Healthcare Research and Quality as part of the Healthcare Cost and Utilization Project. ${ }^{19}$ The NIS is the largest all-payer inpatient-care database, and contains a $20 \%$ stratified sample of yearly discharge data from shortterm, non-Federal, nonrehabilitation hospitals. Data are 
stratified according to geographic region, location (urban/ rural), teaching status, ownership, and hospital bed number. Each hospitalization is treated as an individual entry in the database (ie, individual patients who are hospitalized multiple times may be present in the NIS multiple times). The NIS includes demographic variables, diagnoses, procedures, LOS, and hospital charges. Sample weights are provided to allow for the generation of national estimates, along with information necessary to calculate the variance of estimates.

We utilized the 2012 NIS subset, the most recent year available at the time of data analysis. The 2012 NIS subset contained administrative data from over 7 million hospitalizations, representing more than 4000 hospitals, 44 states, and $95 \%$ of the US population. We excluded patients under 18 years of age and patients with end-stage renal disease (ESRD). We identified patients with ESRD using diagnosis codes and procedure codes from the International Classification of Diseases, Ninth Revision, Clinical Modification (ICD-9-CM, Supplemental Table 1). We also excluded hospitalizations with an ICD-9 diagnosis or procedure code for dialysis but without a diagnosis code for AKI, assuming that these patients were treated with dialysis for ESRD. We and others have used this approach, ${ }^{11,20,21}$ which has been shown to produce high sensitivity and specificity, as well as high positive and negative predictive values (all equal to or greater than 90\%) for differentiating dialysis-requiring AKI (AKI-D) from chronic dialysis. ${ }^{21}$

\section{Primary and Secondary Exposures}

Episodes of AKI were identified using the ICD-9 diagnosis code 584.x. This administrative code for AKI has low sensitivity, but high specificity of approximately $99 \%$ : our cohort includes few false positives, and identifies a more severe spectrum of AKI compared to serum creatinine criteria. ${ }^{21,22}$ For example, the median $\left(25^{\text {th }}, 75^{\text {th }}\right.$ percentile $)$ change in serum creatinine from baseline is estimated at $1.2(0.7$ to 2.1) $\mathrm{mg} / \mathrm{dL}$ compared with $0.2(0.1$ to 0.2$) \mathrm{mg} / \mathrm{dL}$ for patients without an administrative code for AKI. ${ }^{21}$ We defined AKI-D as the presence of an AKI diagnosis code and a diagnosis or procedure code for dialysis. This algorithm for AKI-D has been shown to yield high sensitivity and specificity. ${ }^{21}$ Secondary exposures included several acute medical conditions (myocardial infarction, stroke, venous thromboembolic disease, gastrointestinal bleed, acute pancreatitis, sepsis, and pneumonia) whose incremental costs and LOS could be compared to AKI (Supplemental Table 1).

\section{Covariates}

We assessed patient comorbidities from the 25 diagnoses listed in the NIS for each record (Supplemental Table 1). Hospital-level variables included geographic region, bed number, and teaching status using predetermined NIS definitions. ${ }^{19}$

\section{Outcomes}

The primary outcome was the inpatient cost of each hospital record in 2012 dollars. We estimated costs from the total

\section{TABLE 1. Characteristics of the Cohort}

\begin{tabular}{|c|c|c|c|}
\hline & No AKI & $\mathrm{AKl}^{\mathrm{a}}$ & AKI-D \\
\hline Characteristic, \% & $(n=26,732,623)$ & $n=3,031,026)$ & $(n=106,515)$ \\
\hline Age, mean (SD) & $55.8(0.1)$ & $69.0(0.1)$ & $63.3(0.2)$ \\
\hline \multicolumn{4}{|l|}{ Sex } \\
\hline Male & $38.9 \%$ & $52.8 \%$ & $58.2 \%$ \\
\hline Female & $61.1 \%$ & $47.3 \%$ & $41.8 \%$ \\
\hline \multicolumn{4}{|l|}{ Hospital teaching status } \\
\hline Rural & $12.1 \%$ & $9.8 \%$ & $5.2 \%$ \\
\hline Urban nonteaching & $38.7 \%$ & $38.8 \%$ & $37.0 \%$ \\
\hline Urban teaching & $49.2 \%$ & $51.4 \%$ & $57.8 \%$ \\
\hline \multicolumn{4}{|l|}{$\underline{\text { Hospital region }}$} \\
\hline Northeast & $19.7 \%$ & $18.8 \%$ & $16.1 \%$ \\
\hline West & $18.9 \%$ & $18.4 \%$ & $21.3 \%$ \\
\hline Midwest & $22.9 \%$ & $22.7 \%$ & $22.4 \%$ \\
\hline South & $38.5 \%$ & $40.1 \%$ & $40.2 \%$ \\
\hline \multicolumn{4}{|l|}{ Hospital bed number } \\
\hline Small & $14.5 \%$ & $12.8 \%$ & $9.2 \%$ \\
\hline Medium & $26.5 \%$ & $26.5 \%$ & $24.2 \%$ \\
\hline Large & $59.1 \%$ & $60.7 \%$ & $66.7 \%$ \\
\hline \multicolumn{4}{|l|}{ Acute medical conditions } \\
\hline Myocardial infarction & $2.6 \%$ & $6.7 \%$ & $11.0 \%$ \\
\hline Stroke & $3.0 \%$ & $3.4 \%$ & $4.1 \%$ \\
\hline Venous thromboembolic disease & $2.1 \%$ & $3.9 \%$ & $7.3 \%$ \\
\hline Gastrointestinal bleed & $2.2 \%$ & $5.3 \%$ & $8.9 \%$ \\
\hline Acute pancreatitis & $1.3 \%$ & $1.9 \%$ & $4.4 \%$ \\
\hline Sepsis & $3.6 \%$ & $20.2 \%$ & $43.0 \%$ \\
\hline Pneumonia & $6.6 \%$ & $16.1 \%$ & $27.0 \%$ \\
\hline \multicolumn{4}{|l|}{ Chronic comorbidities } \\
\hline Cancer & $9.0 \%$ & $12.7 \%$ & $14.7 \%$ \\
\hline Chronic kidney disease & $7.1 \%$ & $46.2 \%$ & $51.3 \%$ \\
\hline Congestive heart failure & $11.8 \%$ & $34.0 \%$ & $40.5 \%$ \\
\hline Dementia & $5.4 \%$ & $11.9 \%$ & $3.7 \%$ \\
\hline Diabetes & $21.3 \%$ & $41.6 \%$ & $41.2 \%$ \\
\hline Human immunodeficiency virus & $0.3 \%$ & $0.7 \%$ & $1.0 \%$ \\
\hline Hypertension & $47.4 \%$ & $73.0 \%$ & $66.0 \%$ \\
\hline Chronic obstructive pulmonary disease & $12.7 \%$ & $20.0 \%$ & $18.0 \%$ \\
\hline Peripheral vascular disease & $5.4 \%$ & $10.8 \%$ & $11.6 \%$ \\
\hline \multicolumn{4}{|l|}{ Hospital procedures } \\
\hline Intravenous contrast & $4.9 \%$ & $5.4 \%$ & $8.7 \%$ \\
\hline Blood product transfusion & $6.8 \%$ & $17.8 \%$ & $40.8 \%$ \\
\hline Mechanical ventilation & $2.2 \%$ & $11.2 \%$ & $43.4 \%$ \\
\hline Noninvasive ventilation & $1.5 \%$ & $4.2 \%$ & $8.0 \%$ \\
\hline Cardiopulmonary resuscitation & $0.2 \%$ & $1.3 \%$ & $5.6 \%$ \\
\hline Left ventricular assist device & $0.0 \%$ & $0.1 \%$ & $0.5 \%$ \\
\hline Extracorporeal membrane oxygenation & $0.0 \%$ & $0.1 \%$ & $0.4 \%$ \\
\hline Echocardiogram & $2.3 \%$ & $4.7 \%$ & $8.0 \%$ \\
\hline Coronary angiogram & $4.1 \%$ & $4.7 \%$ & $7.7 \%$ \\
\hline $\begin{array}{l}\text { Percutaneous transluminal coronary } \\
\text { angioplasty }\end{array}$ & $1.8 \%$ & $1.4 \%$ & $2.0 \%$ \\
\hline Cardiopulmonary bypass & $0.8 \%$ & $1.3 \%$ & $3.7 \%$ \\
\hline Coronary artery bypass grafting & $0.6 \%$ & $1.1 \%$ & $2.3 \%$ \\
\hline Heart valve surgery & $0.3 \%$ & $0.7 \%$ & $2.1 \%$ \\
\hline Abdominal aortic aneurysm repair & $0.2 \%$ & $0.2 \%$ & $0.8 \%$ \\
\hline Carotid endarterectomy & $0.4 \%$ & $0.1 \%$ & $0.2 \%$ \\
\hline Peripheral vascular surgery & $0.6 \%$ & $0.8 \%$ & $1.7 \%$ \\
\hline
\end{tabular}




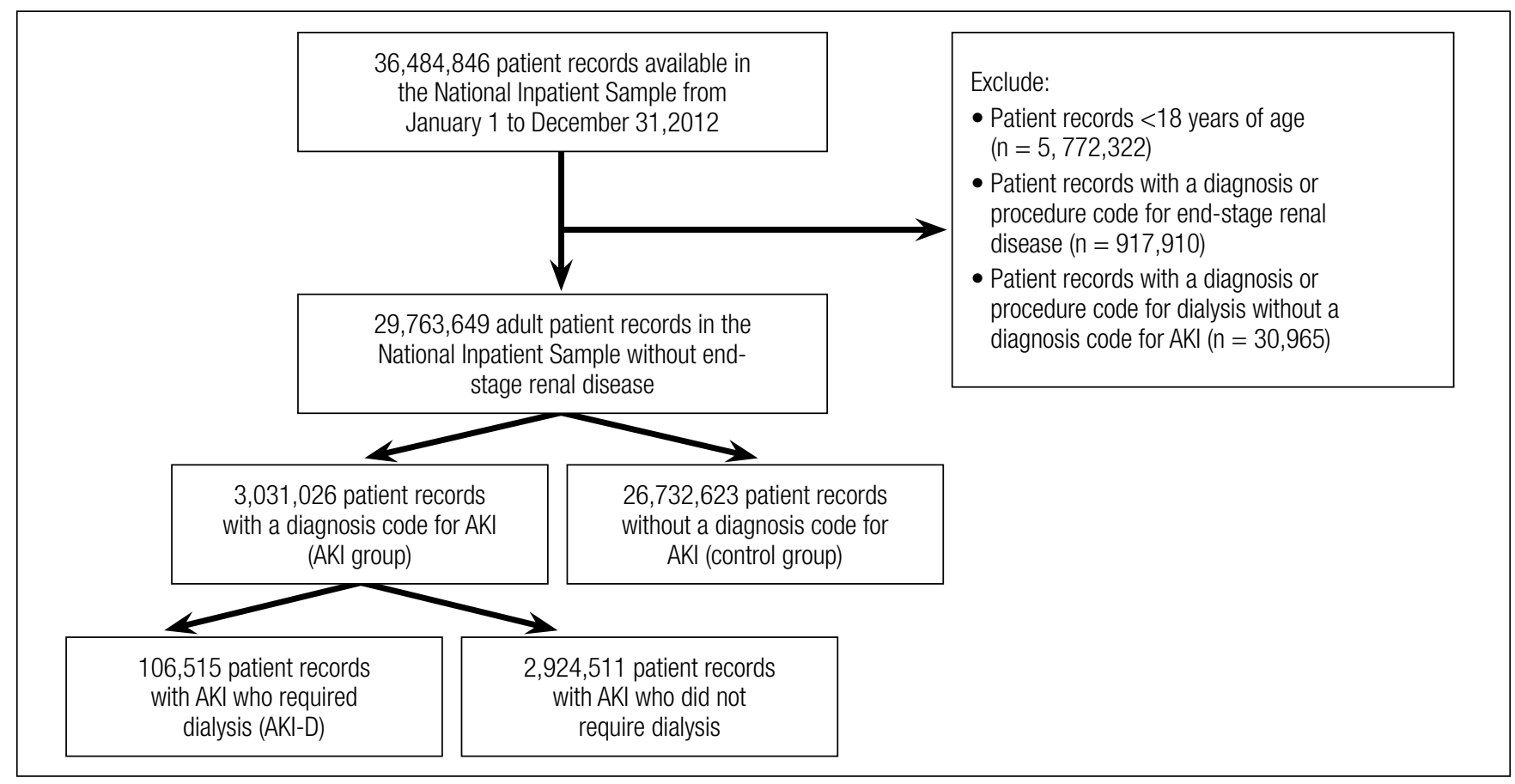

FIG. 1. Inclusion and exclusion criteria used to define a cohort of patients with and without AKI NOTE: Abbreviations: AKI, acute kidney injury; AKI-D, acute kidney injury requiring dialysis.

charge for each hospitalization by applying hospital-specific charge-to-cost ratios. The NIS obtained cost information from the hospital accounting reports collected by the Centers for Medicare and Medicaid Services. ${ }^{19}$ The secondary outcome was hospital LOS.

\section{Statistical Analysis}

We summarized baseline characteristics of the study participants using descriptive statistics. Normally distributed continuous variables were expressed as mean (standard deviation [SD]), and nonparametric continuous variables were expressed as median (25th, 75 th percentile). Categorical variables were expressed as proportions. We calculated the mean increase in cost and LOS of each hospital record, comparing hospital records with AKI and AKI-D to hospital records without AKI. We took the same approach when examining incremental costs and LOS associated with other acute medical conditions. Due to the skewness of cost and LOS data, we used a generalized linear model with a gamma distribution and a log link fitted to the primary or secondary exposure to obtain the unadjusted mean increase in cost and LOS. ${ }^{23,24}$ We incorporated demographics, hospital differences, comorbidities (including AKI when it was compared to the other acute medical conditions), and procedures into the generalized linear model to calculate the adjusted mean increase in cost and LOS. This method also provides the adjusted percentage change in hospital costs and LOS from the estimated beta-coefficients in the multivariable model. We calculated the proportion of variation in the outcomes explained by the generalized linear models using pseudo R-squared measured by the Kullback-Leibler divergence. ${ }^{25}$ As a companion analy- sis, we repeated estimates for AKI-D when dialysis was initiated within 7 days of hospital admission because subsequent events during the hospital stay would more likely be attributable to the AKI episode. All analyses presented account for the NIS survey design (weighting and stratification) and subpopulation measurements to generate national estimates. We created the cohort using the Statistical Analysis System software, version 9.4 (SAS Institute, Cary, North Carolina) and conducted the analyses using StataMP, version 14.0 (Stata Corporation, College Station, Texas).

\section{RESULTS}

\section{Patient Characteristics}

Between January 1 and December 31, 2012, there were $36,484,846$ hospitalization records available in the NIS; 948,875 adult records $(2.6 \%)$ were classified as having ESRD and 29,763,649 (81.6\%) were included in the final cohort. Within the final cohort, 3,031,026 (10.2\%) hospitalizations were complicated by AKI, of which 106,515 (3.5\%) required dialysis (corresponding to $0.36 \%$ of the analytic cohort) (Figure 1).

Compared to patients without AKI, patients with AKI were older (69.0 years vs. 55.8 years) and a larger proportion were male $(52.8 \%$ vs. $38.9 \%)$. All measured comorbidities were more prevalent in patients with AKI. Patients with AKI also underwent more hospital procedures than patients without AKI (Table 1).

\section{Hospitalization Costs}

Figures $2 \mathrm{~A}$ and $2 \mathrm{~B}$ show unadjusted and multivariable-adjusted mean increases in cost of a hospitalization with AKI 

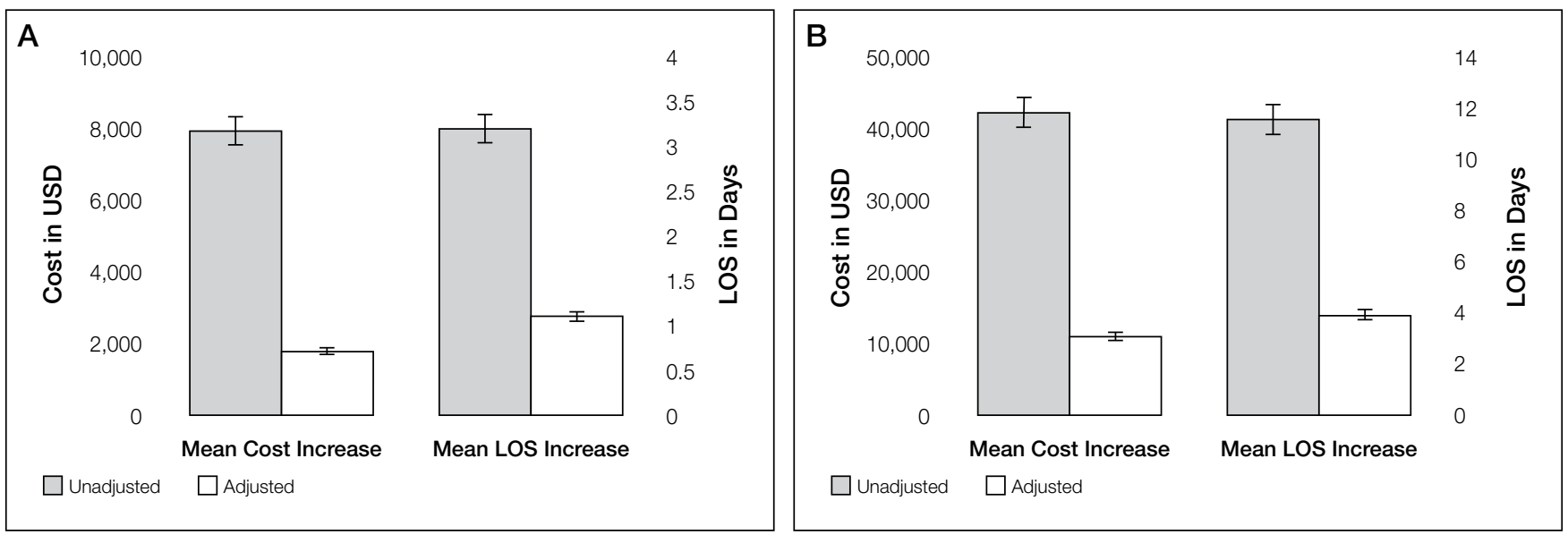

FIG. 2. (A) Mean increase in cost and LOS per hospital admission of AKI compared to a hospital admission without AKI. Costs increases are in 2012 dollars. White bars are adjusted for the demographic factors, hospital differences, comorbidities, and procedures listed in Table 1. Error bars represent the $95 \%$ confidence intervals. The multivariable model explained $67 \%$ of the variation in total hospital costs and $47 \%$ of the variation in LOS. (B) Mean increase in cost and LOS per hospital admission of AKI-D compared to a hospital admission without AKI. Cost increases are in 2012 dollars. White bars are adjusted for the demographic factors, hospital differences, comorbidities, and procedures listed in Table 1. Error bars represent the $95 \%$ confidence intervals. The multivariable model explained $53 \%$ of the variation in total hospital costs and $64 \%$ of the variation in LOS.

NOTE: Abbreviations: AKI, acute kidney injury; AKI-D, acute kidney injury requiring dialysis; LOS, length of stay.

and AKI-D compared to a hospitalization without AKI. Extrapolating to the 2012 population estimates in Table 1 for AKI and AKI-D, increases in cost related to AKI ranged from $\$ 24.0$ billion (unadjusted) to $\$ 5.4$ billion (adjusted) and for AKI-D ranged from $\$ 4.5$ billion (unadjusted) to $\$ 1.2$ billion (adjusted).

Mean increases in the cost of a hospitalization for AKI exceeded costs associated with other acute medical conditions such as myocardial infarction and gastrointestinal bleeding. Costs associated with AKI were similar to hospitalizations for stroke, acute pancreatitis, and pneumonia. Costs of AKI-D exceeded those related to sepsis and venous thromboembolic disease (Table 2). AKI was the most common of the acute medical conditions examined (3,031,026 patients, 10.2\%).

Major drivers of cost included urban and teaching hos- pitals, hospitals in the Southern US (relative to other regions), hospitals with a larger number of beds, most acute medical conditions, cancer, and hospital procedures. Older age was associated with higher costs with non-AKI hospitalizations but lower costs with AKI hospitalizations (0.67\% vs. $-0.44 \%$, per year of age). Determinants of hospital costs are shown in Supplemental Table 2. Generally, hospital procedures accounted for the largest relative increases in cost.

\section{Length of Stay}

Figures $2 \mathrm{~A}$ and $2 \mathrm{~B}$ show unadjusted and multivariable-adjusted mean increases in LOS for a hospitalization with AKI and AKI-D compared to a hospitalization without AKI. Extrapolating to the 2012 population estimates in Table 1 for AKI and AKI-D, increases in LOS related to AKI ranged

\section{TABLE 2. Mean Increase in Cost and LOS per Hospital Admission of AKI and Other Acute Medical Conditions}

\begin{tabular}{lccc}
\hline Acute Medical Condition & Prevalence, No. (\%) & $\begin{array}{c}\text { Adjusted Mean Cost } \\
\text { Increase in 2012 US Dollars (95\% Cl) }\end{array}$ & $\begin{array}{c}\text { Adjusted Mean Length of Stay } \\
\text { Increase in Days (95\% Cl) }\end{array}$ \\
\hline AKI & $3,031,026(10.2)$ & $1795(1692,1899)$ & $1.1(1.1,1.1)$ \\
\hline AKI requiring dialysis (AKI-D) & $106,515(0.4)$ & $11016(10468,11564)$ & $3.9(3.8,4.1)$ \\
\hline Myocardial infarction & $901,276(3.0)$ & $14(-91,119)$ & $0.1(0.1,0.2)$ \\
\hline Stroke & $901,227(3.0)$ & $1427(1281,1573)$ & $0.1(0,0.1)$ \\
\hline Venous thromboembolic disease & $677,202(2.3)$ & $3782(3611,3953)$ & $2.3(2.2,2.3)$ \\
\hline Gastrointestinal bleed & $743,692(2.5)$ & $-860(-961,-759)$ & $0(0,0.1)$ \\
\hline Acute pancreatitis & $413,827(1.4)$ & $1802(1676,1929)$ & $1.1(1.1,1.2)$ \\
\hline Sepsis & $1,577,242(5.3)$ & $4882(4696,5068)$ & $2.1(2.1,2.2)$ \\
\hline Pneumonia & $2,246,687(7.5)$ & $1705(1584,1825)$ & $1.2(1.2,1.2)$ \\
\hline
\end{tabular}

aFor each comparison, the reference group is patients without the condition of interest (for AKI-D, the reference group is patients without AKI). All estimates are adjusted for the demographic factors, hospital differences, comorbidities, and procedures listed in Table 1. Non-AKI conditions are also adjusted for AKI.

'DThe AKI group includes patients with AKI-D.

NOTE: Abbreviations: AKI, acute kidney injury; AKI-D, acute kidney injury requiring dialysis; $\mathrm{Cl}$, confidence interval; LOS, length of stay. 
from 9.8 million days (unadjusted) to 3.3 million days (adjusted) and for AKI-D ranged from 1.2 million days (unadjusted) to 0.4 million days (adjusted).

When compared to other acute medical conditions, the mean increase in LOS of an AKI hospitalization resembled the order for mean increases in cost (Table 2). Major drivers of LOS also resembled drivers of costs, with the exception of some common cardiovascular procedures (percutaneous transluminal coronary angioplasty, abdominal aortic aneurysm repair, and carotid endarterectomy) that were associated only with prolonged LOS in the AKI and AKI-D groups (Supplemental Table 3).

\section{Companion Analysis}

In an analysis of 78,220 patients who developed AKI-D within 7 days of hospital admission (73\% of AKI-D cases), increases in cost ranged from $\$ 32,133$ (unadjusted) to $\$ 8594$ (adjusted) and increases in LOS ranged from 8.4 days (unadjusted) to 2.9 days (adjusted) compared to patients without AKI.

\section{DISCUSSION}

We found that hospitalizations complicated by AKI were more costly-between $\$ 1800$ and $\$ 7900$ - than hospitalizations that did not involve AKI, which indicates that AKI could be responsible for billions of dollars of annual healthcare spending. Relative to several other acute medical conditions, AKI was more common and expensive; when AKI was severe enough to require dialysis, costs of $\mathrm{AKI}$ exceeded all other acute medical conditions by a large margin.

Several single-center and regional studies have highlighted the association of AKI with hospital costs and LOS. In a single-center study conducted in the late 1990s, Chertow et $\mathrm{al}^{14}$ described mean cost increases between $\$ 4900$ (adjusted) and $\$ 8900$ (unadjusted) and LOS increases of 3.5 days (adjusted) using serum creatinine criteria to define AKI. ${ }^{14}$ These higher adjusted estimates may result because their multivariable models did not adjust for several major determinants of cost, including several procedures and hospital-level variables. A study at the same academic center in 2010, which adjusted for some procedures, found AKI was associated with a 2.8-day increase in LOS and a \$7082 increase in costs; ${ }^{2}$ however, this study also could not adjust for hospital-level variables because of the single-center design. Fischer et $\mathrm{al}^{15}$ were able to adjust for hospital teaching status in their study that included 23 local hospitals. Similar to our results, teaching hospitals were associated with an approximately $17 \%$ increase in cost compared to nonacademic hospitals. However, this study excluded patients who required critical care or mechanical ventilation, which limits the generalizability of their cost estimates. Another limitation of these 3 studies is that they were all conducted in Massachusetts. Beyond the US, the economic burden of AKI has been studied in England where the annual cost of AKI-related inpatient care has been estimated at $\$ 1.4$ billion. ${ }^{16}$ In addition to incomplete procedure and hospital-level adjustment, this study is limited by its ascertainment of AKI and costs, which was extrapolated from 1 hospital region to the rest of England.

Our study adds to the existing evidence in a number of ways. It uses nationally representative data to determine a lower and an upper limit of increases in cost and LOS attributable to AKI. The adjusted value is likely overly conservative; it minimizes the influence of events that are attributable to AKI and does not account for complications that may be caused by, or otherwise related to, AKI. The unadjusted value is likely an overestimate, attributing events during an AKI hospitalization to the AKI episode, even if they precede $\mathrm{AKI}$. In clinical practice, most patients fall between these 2 extremes. Therefore, we suggest using the adjusted and unadjusted estimates to provide a range of the cost and LOS increases that are attributable to AKI. This interpretation is also supported by the companion analysis that minimizes the effect of pre-AKI events, where the unadjusted cost and LOS estimates for AKI-D occurring early during a hospitalization fell between the unadjusted and adjusted estimates for the main AKI-D analysis. Therefore, our data suggest that each hospitalization complicated by AKI is associated with a cost increase between $\$ 1800$ and $\$ 7900$ and an LOS increase between 1.1 days and 3.2 days. Not surprisingly, the burden of AKI-D was more pronounced with a cost increase between $\$ 11,000$ and $\$ 42,100$ and an LOS increase between 3.9 days and 11.5 days.

Unlike previous studies, these analyses are fully adjusted for procedures and multiple hospital-level variables (such as teaching status, region, and bed number). These adjustments are important because procedures account for much of the incremental cost and LOS associated with AKI, and each hospital-level variable may increase the cost and LOS of an AKI hospitalization by $10 \%$ to $25 \%$ (Supplemental Tables 2 and 3). Even though the relative increases in cost and LOS associated with different comorbidities and procedures were largely similar between patients with and without AKI, the absolute increases were usually larger in patients with AKI rather than without AKI because of their higher baseline estimates. We also observed that each year of age was associated with increased costs in patients without AKI, but decreased costs in patients with AKI. We suspect this difference is due to the lesser (and ultimately less costly) injury required to induce $\mathrm{AKI}$ in elderly patients who have less physiologic reserve. ${ }^{26}$ Moreover, we placed the burden of $\mathrm{AKI}$ in relation to other acute medical conditions, where its total estimated annual costs of $\$ 5.4$ billion were exceeded only by the $\$ 7.7$ billion attributed to sepsis.

Our results emphasize that AKI is an important contributor to hospital costs and LOS. Despite these consequences, there have been very few innovations in the prevention and management of AKI over the last decade. ${ }^{27,28}$ The primary treatment for severe AKI remains dialysis, and recent clinical trials suggest that we may have reached a dose plateau in the value of dialytic therapy. ${ }^{8,29}$ Several opportunities, such as advances in basic science and clinical care, may improve the care of patients with AKI. Translational research chal- 
lenges in AKI have been reviewed, with treatment strategies that include hemodynamic, inflammatory, and regenerative mechanisms. ${ }^{28,} 30$ In a recent report from the National Confidential Enquiry into Patient Outcome and Death in the United Kingdom, 30\% of AKI episodes that occurred inhospital were preventable, and only $50 \%$ of patients with AKI were deemed to have received good care. ${ }^{31}$ Our results suggest that even small progress in these areas could yield significant cost savings. One starting point suggested by our findings is a better understanding of the reasons underlying the association between hospital-level variables and differences in cost and LOS. Notably, there have been few efforts to improve AKI care processes on the same scale as sepsis, ${ }^{32}$ myocardial infarction, ${ }^{33,34}$ stroke, ${ }^{35}$ and venous thromboembolic disease. ${ }^{36}$

Strengths of this study include cost and LOS estimates of AKI from different hospitals across the US, including academic and community institutions. As a result, our study is significantly larger and more representative of the US population than previously published studies. Moreover, we utilized data from 2012, which accounts for the increasing incidence of AKI and recent advances in critical care medicine. We were also able to adjust for comorbid conditions, procedures, severity of illness, and hospital-level variables, which provide a conservative lower limit of the burden of AKI on hospitalized patients.

Our study has limitations. First, we used administrative codes to identify patients with AKI. The low sensitivity of these codes suggests that many patients with milder forms of AKI were probably not coded as such. Accordingly, our findings should be generally applicable to patients with moderate to severe AKI rather than to those with mild AKI. ${ }^{21,22} \mathrm{Sec}$ ond, the NIS lacks granularity on the details and sequence of events during a hospitalization. As a result, we could not determine the timing of an AKI episode during a hospitalization or whether a diagnosis or procedure was the cause or consequence of an AKI episode (ie, day 1 as the reason for admission vs. day 20 as a complication of surgery). Both the timing and cause of an AKI episode may influence cost and LOS, which should be considered when applying our results to patient care. We did not attempt to estimate the costs associated with comorbidities such as congestive heart failure and chronic obstructive pulmonary disease because we could not determine the acuity of disease in the NIS. Third, despite our efforts, residual confounding is likely, especially since administrative data limit our ability to capture the severity of comorbid conditions and the underlying illness. Fourth, the NIS does not contain individual patient identifiers, so multiple hospitalizations from the same patient may be represented.

Even our most conservative estimates still attribute $\$ 5.4$ billion and 3.3 million hospital-days to AKI in 2012. These findings highlight the need for hospitals, policymakers, and researchers to recognize the economic burden of AKI. Future work should focus on understanding hospital-level differences in AKI care and the effect on patient morbidity and mortality. National and hospital-wide quality improvement programs are also needed. Such initiatives have commenced in the United Kingdom, ${ }^{37}$ and similar efforts are needed in North America to develop and coordinate cost-effective strategies to care for patients with AKI.

Disclosures: Samuel A. Silver, MD, MSc, is supported by a Kidney Research Scientist Core Education and National Training Program Post-Doctoral Fellowship (co-funded by the Kidney Foundation of Canada, Canadian Society of Nephrology, and Canadian Institutes of Health Research). Glenn M. Chertow, MD, MPH, is supported by a K24 mid-career mentoring award from NIDDK (K24 DK085446). These funders had no role in the design and conduct of the study; collection, management, analysis, and interpretation of the data; preparation, or approval of the manuscript; or decision to submit the manuscript for publication. The authors report no financial conflicts of interest.

\section{References}

1. Waikar SS, Liu KD, Chertow GM. Diagnosis, epidemiology and outcomes of acute kidney injury. Clin J Am Soc Nephrol. 2008;3:844-861.

2. Zeng X, McMahon GM, Brunelli SM, Bates DW, Waikar SS. Incidence, outcomes, and comparisons across definitions of AKI in hospitalized individuals. Clin J Am Soc Nephrol. 2014;9:12-20.

3. Susantitaphong P, Cruz DN, Cerda J, et al. Acute Kidney Injury Advisory Group of the American Society of Nephrology. World incidence of AKI: a meta-analysis. Clin J Am Soc Nephrol. 2013;8:1482-1493.

4. Dasta JF, Kane-Gill SL, Durtschi AJ, Pathak DS, Kellum JA. Costs and outcomes of acute kidney injury (AKI) following cardiac surgery. Nephrol Dial Transplant. 2008;23:1970-1974.

5. Hobson C, Ozrazgat-Baslanti T, Kuxhausen A, et al. Cost and mortality associated with postoperative acute kidney injury. Ann Surg. 2015;261:1207-1214.

6. Vieira JM Jr, Castro I, Curvello-Neto A, et al. Effect of acute kidney injury on weaning from mechanical ventilation in critically ill patients. Crit Care Med. 2007;35:184-191

7. Selby NM, Kolhe NV, McIntyre CW, et al. Defining the cause of death in hospitalised patients with acute kidney injury. PLoS One. 2012;7:e48580.

8. Palevsky PM, Zhang JH, O'Connor TZ, et al. Intensity of renal support in critically ill patients with acute kidney injury. N Engl J Med. 2008;359(1):7-20.

9. Uchino S, Bellomo R, Morimatsu H, et al. Continuous renal replacement therapy: a worldwide practice survey. The beginning and ending supportive therapy for the kidney (B.E.S.T. kidney) investigators. Intensive Care Med. 2007;33:1563-1570.

10. Siew ED, Davenport $A$. The growth of acute kidney injury: a rising tide or just closer attention to detail? Kidney Int. 2015;87:46-61.

11. Hsu RK, McCulloch CE, Dudley RA, Lo LJ, Hsu CY. Temporal changes in incidence of dialysis-requiring AKI. J Am Soc Nephrol. 2013;24:37-42.

12. Xue JL, Daniels F, Star RA, et al. Incidence and mortality of acute renal failure in Medicare beneficiaries, 1992 to 2001. J Am Soc Nephrol. 2006;17:1135-1142.

13. Saran R, Li Y, Robinson B, et al. US Renal Data System 2015 annual data report: epidemiology of kidney disease in the United States. Am J Kidney Dis. 2016;67(3 suppl 1):S1-S434.

14. Chertow GM, Burdick E, Honour M, Bonventre JV, Bates DW. Acute kidney injury, mortality, length of stay, and costs in hospitalized patients. J Am Soc Nephrol. 2005; 16:3365-3370.

15. Fischer MJ, Brimhall BB, Lezotte DC, Glazner JE, Parikh CR. Uncomplicated acute renal failure and hospital resource utilization: a retrospective multicenter analysis. Am J Kidney Dis. 2005;46:1049-1057.

16. Kerr M, Bedford M, Matthews B, O'Donoghue D. The economic impact of acute kidney injury in England. Nephrol Dial Transplant. 2014;29:1362-1368.

17. De Smedt DM, Elseviers MM, Lins RL, Annemans L. Economic evaluation of different treatment modalities in acute kidney injury. Nephrol Dial Transplant. 2012;27:4095-5101.

18. Srisawat N, Lawsin L, Uchino S, Bellomo R, Kellum JA; BEST Kidney Investigators. Cost of acute renal replacement therapy in the intensive care unit: results from The Beginning and Ending Supportive Therapy for the Kidney (BEST Kidney) study. Crit Care. 2010;14:R46.

19. Agency for Healthcare Research and Quality. Healthcare Cost and Utilization Project (HCUP). Overview of the National (Nationwide) Inpatient Sample (NIS). Available at: http://www.hcup-us.ahrq.gov/nisoverview.jsp. Accessed January 10, 2016.

20. Lenihan CR, Montez-Rath ME, Mora Mangano CT, Chertow GM, Winkelmayer WC. Trends in acute kidney injury, associated use of dialysis, and mortality after 
cardiac surgery, 1999 to 2008. Ann Thorac Surg. 2013;95:20-28.

21. Waikar SS, Wald R, Chertow GM, et al. Validity of international classification of diseases, ninth revision, clinical modification codes for acute renal failure. J Am Soc Nephrol. 2006;17:1688-1694.

22. Grams ME, Waikar SS, MacMahon B, Whelton S, Ballew SH, Coresh J. Performance and limitations of administrative data in the identification of AKI. Clin J Am Soc Nephrol. 2014;9:682-689.

23. Blough DK, Madden CW, Hornbrook MC. Modeling risk using generalized linear models. J Health Econ. 1999;18:153-171.

24. Malehi AS, Pourmotahari F, Angali KA. Statistical models for the analysis of skewed healthcare cost data: a simulation study. Health Econ Rev. 2015;5:11.

25. Cameron AC, Windmeijer FA. An R-squared measure of goodness of fit for some common nonlinear regression models. J Econometrics. 1997(77):329-342.

26. Coca SG. Acute kidney injury in elderly persons. Am J Kidney Dis. 2010;56:122-131.

27. Bonventre JV, Basile D, Liu KD, et al; Kidney Research National Dialogue (KRND). AKI: a path forward. Clin J Am Soc Nephrol. 2013;8:1606-1608.

28. Okusa MD, Rosner MH, Kellum JA, Ronco C; Acute Dialysis Quality Initiative XIII Workgroup. Therapeutic targets of human AKI: harmonizing human and animal AKI. J Am Soc Nephrol. 2016;27:44-48.

29. Pannu N, Klarenbach S, Wiebe N, Manns B, Tonelli M; Alberta Kidney Disease Network. Renal replacement therapy in patients with acute renal failure: a systematic review. JAMA. 2008;299:793-805.

30. Silver SA, Cardinal H, Colwell K, Burger D, Dickhout JG. Acute kidney injury: preclinical innovations, challenges, and opportunities for translation. Can J Kid- ney Health Dis. 2015;2:30

31. Stewart J, Findlay G, Smith N, Kelly K, Mason M. Adding insult to injury: a review of the care of patients who died in hospital with a primary diagnosis of acute kidney injury (acute renal failure). A report by the National Confidential Enquiry into Patient Outcome and Death 2009. Available at: http://www.ncepod. org.uk/2009aki.html. Accessed April 4, 2016.

32. Society of Critical Care Medicine. Surviving Sepsis Campaign. Available at: http://www.survivingsepsis.org/Pages/default.aspx. Accessed April 3, 2016.

33. Mehta RH, Montoye CK, Gallogly M, et al; GAP Steering Committee of the American College of Cardiology. Improving quality of care for acute myocardial infarction: The Guidelines Applied in Practice (GAP) Initiative. JAMA. 2002;287:1269-1276.

34. Lewis WR, Peterson ED, Cannon CP, et al. An organized approach to improvement in guideline adherence for acute myocardial infarction: results with the Get With The Guidelines quality improvement program. Arch Intern Med. 2008;168:1813-1819.

35. Schwamm LH, Fonarow GC, Reeves MJ, et al. Get With the Guidelines-stroke is associated with sustained improvement in care for patients hospitalized with acute stroke or transient ischemic attack. Circulation. 2009;119:107-115.

36. Maynard G. Preventing Hospital-associated Venous Thromboembolism: A Guide for Effective Quality Improvement. $2^{\text {nd }}$ ed. Rockville, MD: Agency for Healthcare Research and Quality; October 2015. AHRQ Publication No. 16-0001-EF.

37. NHS England: Acute kidney injury programme. Available at: http://www. Thinkkidneys.nhs.uk. Accessed April 3, 2016. 